\title{
QUÉ MÚSICA ENSEÑAR HOY: DEL PARADIGMA DE APRECIACIÓN MUSICAL A LA SOCIEDAD DEL SIGLO XXI
}

\author{
Susana Flores \\ Universidad de La Rioja
}

RESUMEN: En España la música forma parte de la educación general, tanto en la etapa de primaria como en la de secundaria. Pese a la juventud de esta área curricular, ha sido una de las asignaturas que más ha evolucionado en los últimos cuarenta años, pasando de un enfoque tradicional a reflejar los cambios tecnológicos y la realidad de la sociedad actual.

Palabras clave: Educación musical en España, legislación educativa, currículo, educación primaria, educación secundaria.

\section{WHAT KIND OF MUSIC TEACHING TODAY: FROM PARADIGM OF MUSICAL APPRECIATION TO XXI CENTURY SOCIETY}

\begin{abstract}
In Spain the music is part of the general education, both during the primary and secondary education. Despite the youth of this area of the curriculum, it has been one of the subjects with a greater development in the past forty years, from a traditional approach to reflect changes in technology and the reality of today's society.
\end{abstract}

Keywords: Spanish music education, Educational laws, curriculum, primary education, secondary education.

En 2002 entraba en vigor en Estados Unidos la Ley No Child Left Behind (Que ningún niño se quede atrás), que compensaba a los colegios que obtenían mejores resultados en asignaturas troncales como lengua, matemáticas o ciencias, y sancionaba a los que quedaban detrás con la pérdida de la subvención 
económica. Probablemente, la intención del gobierno no era empobrecer las enseñanzas artísticas en Estados Unidos, pero el resultado fue que en 2006, a fin de concentrar sus esfuerzos en las materias objeto de examen, más del $70 \%$ de los colegios habían reducido sus currículos prescindiendo de las asignaturas artísticas.

\section{Por qué es necesario enseñar música}

En líneas generales apenas existen dudas de por qué algunas asignaturas deben formar parte de la enseñanza general; sin embargo, en el caso de la música, el profesorado a menudo se ve obligado a justificar su presencia en el currículo. Por supuesto que para alguien que aprecia y valora la música, ésta tiene un valor único e irreemplazable, si bien, como explica Ross (2012) es difícil traducir esta convicción a datos concluyentes.

Con frecuencia podemos encontrar justificaciones relacionadas con el desarrollo neurológico. Parece demostrado que el ser humano está biológicamente dotado para ser musical, y que esta predisposición genética para la musicalidad no solo tiene consecuencias artísticas, sino también emocionales y sociales. Para mostrar la repercusión del aprendizaje de música en el desarrollo del cerebro, a menudo se han comparado los efectos de estas prácticas en individuos con formación musical y sin ella. Los resultados obtenidos muestran que en los niños que se inician pronto en el aprendizaje de música el desarrollo cerebral es distinto al de aquellos que no reciben formación, y que cuando llegan a adultos tienen unas repuestas más fuertes y rápidas ante los estímulos musicales (Hodges 2006). Entre esas diferencias destaca un cuerpo calloso ${ }^{1}$ más extenso, la gran simetría de la corteza motora ${ }^{2}$ de los dos hemisferios, y diferencias en el tamaño del cerebelo y en algunas regiones de la materia gris (Schlaug 2003). Estas características podrían reflejar la necesidad de una mayor comunicación entre ambos hemisferios, el desarrollo de habilidades motoras para la utilización de ambas manos, y la necesidad de integrar habilidades cognitivas y emocionales (Hallam 2006).

El desarrollo del cerebro musical es paralelo al del cerebro general y las habilidades musicales que se reflejan en él parecen estar influenciadas tanto por la información genética como por las experiencias de aprendizaje, ya que el cerebro musical es altamente plástico. Estudios como los de Stewart et al. (2003) muestran que el aprendizaje de la lectura musical activa un área del cerebro específica, y que después de un período de tiempo la notación musical se automatiza y en el caso de los instrumentistas se traduce en una respuesta motora.

1. El cuerpo calloso es la parte que conecta ambos hemisferios.

2. El córtex motor o corteza motora, es un área del cerebro encargada del funcionamiento motor de los miembros (dedos, manos, brazos, etc.) y los demás órganos motores. 
En los años noventa un grupo de investigadores analizaba los resultados de un test de razonamiento espacio-temporal en una muestra de estudiantes que habían sido expuestos durante diez minutos a la audición de la Sonata K. 448 de Mozart (Rauscher, Shaw y Ky 1993). Las observaciones permitían comprobar que los alumnos que habían escuchado esta música obtenían mejores resultados que aquellos que habían participado en actividades de relajación o que simplemente habían permanecido en silencio. El efecto era pequeño y poco duradero, sin embargo, la investigación obtuvo una gran repercusión y los medios de comunicación acuñaron la denominación de "Efecto Mozart", interpretando que la audición pasiva de la música de este compositor contribuía a fomentar la inteligencia sin esfuerzo.

Posteriores investigaciones mostraron lo engañoso de este término, primero porque no solo la música de Mozart permitía conseguir estos resultados, ya que éstos también se apreciaban con la audición de otros compositores y de otros estilos musicales o incluso con otras actividades; sino porque lo que realmente había sucedido durante los diez minutos de exposición a la música era una estimulación y una modificación en el estado de ánimo, algo que a su vez también afectaba a la cognición (Schellenberg, 2005).

Sea como fuere, la controversia en torno al "Efecto Mozart" renovó el interés por la investigación sobre los efectos de la música en la cognición, y si bien se demostró que el efecto de una audición musical producía un pequeño y efímero estímulo, que no obstante permitía ser constatado en tests de razonamiento, posteriormente se ha podido comprobar que los alumnos mejoraban ligeramente su nivel general de inteligencia si habían sido expuestos al aprendizaje musical (Schellenberg, 2004).

Otras justificaciones tienen que ver con el desarrollo de la inteligencia. En los años ochenta Gardner (1983) publicaba Multiple intelligences, un libro en el que se posicionaba en contra de la concepción unitaria de inteligencia y proponía la existencia de siete tipos de inteligencia, que años más tarde ampliaría a ocho. Precisamente, una de las facultades del modelo de Gardner era la inteligencia musical, que supone la capacidad de interpretar, componer y apreciar pautas musicales, y es comparable, estructuralmente hablando, a la inteligencia lingüística. Por ese motivo, el autor consideraba que carece de sentido Ilamar inteligencia al potencial lingüístico y talento al musical. Para Gardner las inteligencias no son algo que se pueda ver o contar, sino que son potenciales que se activan o no en función de las oportunidades que se le brinden al individuo. Asimismo, el psicólogo consideraba que si bien las inteligencias son relativamente independientes entre sí, datos empíricos indicaban la vinculación entre algunas inteligencias. Por todo ello, la posibilidad de que la educación general ofrezca al alumnado la oportunidad de tomar contacto con la experiencia musical todavía adquiriría una mayor importancia. 
Años más tarde, Schellenberg (2005) intentaba explicar qué aspectos del aprendizaje musical podían desencadenar el desarrollo general de la inteligencia, concluyendo que no solo se trataría de herramientas de aprendizaje como la atención, la práctica, la lectura de notación, la memorización de pasajes musicales, el aprendizaje de estructuras musicales, el desarrollo de habilidades técnicas o la expresión de emociones, entre otras muchas, ya que estas herramientas también podrían estar presentes en otras áreas; sino que a todo ello se añadía el hecho de que la música es un arte abstracto, lo que contribuiría al desarrollo de este tipo de razonamiento en general, y permitiría en parte explicar el incremento del nivel de inteligencia. Pero además, para Schellenberg el aprendizaje de música presentaría similitudes con el aprendizaje de una segunda lengua, lo que al igual que el bilingüismo conferiría importantes beneficios en el desarrollo cognitivo.

\section{La educación obligatoria en España durante el siglo XX}

En menos de un siglo el sistema educativo español ha experimentado una gran evolución. Pero indudablemente, uno de los cambios más significativos, y reflejo de las mejoras sociales y económicas que se han ido logrando a lo largo del siglo $\mathrm{XX}$, ha sido el de la obligatoriedad y gratuidad en la educación, que ha pasado de tres cursos con la Ley de Instrucción Pública con la iniciábamos el siglo XX, hasta los diez cursos en la actualidad. De este modo, hoy día una parte de la educación secundaria forma parte de la educación básica y tiene carácter obligatorio.

$\mathrm{Si}$ bien los sistemas educativos que se han sucedido a lo largo del siglo XX son de sumo interés, en nuestro caso sólo vamos a ocuparnos de aquéllos en los que el área de música existe como asignatura propia de la educación general, algo que no sucedió hasta la Ley General de Educación de 1970. Es cierto que en el Plan de Bachillerato de 1938 la música se mencionaba en el bloque de Educación física, artística y patriótica, si bien todavía no se planteaba como asignatura con un currículo propio.

Con la Ley General de Educación de 1970 la estructura del sistema educativo se modificaba sustancialmente, y la Educación Primaria, de carácter obligatorio y gratuito, se extendía durante los ocho cursos de Enseñanza General Básica (EGB), mientras que la Secundaria era de carácter voluntario y se estructuraba en tres cursos de Bachillerato (BUP) que pretendían aunar lo cultural y lo científico, y un Curso de Orientación Universitaria (COU).

La Ley de Ordenación General del Sistema Educativo (LOGSE) de 1990 modificaba de nuevo la duración de la educación obligatoria, afectando sobre todo a la estructura de la educación secundaria. Una de las innovaciones de esta nueva ley eran los nuevos límites para esta etapa, que por primera tenía una parte obligatoria. Su duración se extendía desde los 12 años de edad (dos cursos antes que el sistema educativa anterior) hasta los 16 (dos años más tarde). 
Con esta nueva estructura la Educación Secundaria Obligatoria (ESO) pasaba a durar cuatro cursos divididos en dos ciclos de dos cursos cada uno. La Tabla 1 muestra el cambio producido en la obligatoriedad de la educación reflejando la enseñanza obligatoria en la parte sombreada.

Tabla 1. Estructura de la Educación Primaria y Secundaria en la LGE y la LOGSE.

\begin{tabular}{|c|c|}
\hline Ley General de $\mathbf{1 9 7 0}$ & LOGSE \\
\hline $1^{\circ}$ EGB & $1^{\circ}$ Primaria \\
$2^{\circ}$ EGB & $2^{\circ}$ Primaria \\
$3^{\circ}$ EGB & $3^{\circ}$ Primaria \\
$4^{\circ}$ EGB & $4^{\circ}$ Primaria \\
$5^{\circ} \mathrm{EGB}$ & $5^{\circ}$ Primaria \\
$6^{\circ} \mathrm{EGB}$ & $6^{\circ}$ Primaria \\
\hline $7^{\circ} \mathrm{EGB}$ & $1^{\circ} \mathrm{ESO}$ \\
$8^{\circ} \mathrm{EGB}$ & $2^{\circ} \mathrm{ESO}$ \\
\hline $1^{\circ} \mathrm{BUP}$ & $3^{\circ} \mathrm{ESO}$ \\
$2^{\circ} \mathrm{BUP}$ & $4^{\circ} \mathrm{ESO}$ \\
\hline $3^{\circ} \mathrm{BUP}$ & $1^{\circ}$ Bachillerato \\
\hline COU & $2^{\circ}$ Bachillerato \\
\hline
\end{tabular}

La estructura que inauguraba la LOGSE se ha mantenido durante las dos siguientes leyes de educación. Tanto la Ley Orgánica de Calidad en la Educación (LOCE) de 2002, que no llegó a aplicarse, como la Ley Orgánica de Educación (LOE) de 2006 han conservado la disposición de cuatro cursos para la Educación Secundaria Obligatoria que implantaba la LOGSE, si bien con algunas modificaciones.

\section{La música en la educación española}

Como hemos indicado anteriormente, la asignatura de música forma parte del currículo de educación general española desde la Ley General de 1970. Sin embargo, y pese a ser una asignatura de reciente creación, tanto sus contenidos como el planteamiento general de la propia ley han ido evolucionando a través de las diferentes legislaciones.

Con la Ley General de Educación de 1970 la asignatura de música comenzó a impartirse en la educación primaria y secundaria. En el primer caso la asignatura no tenía un enfoque independiente, sino que estaba integrada con otras 
materias como plástica o educación física en un área denominada Expresión Dinámica. Sin embargo, en el caso de la educación secundaria, la asignatura recibía un trato más específico, y bajo la denominación de "Música y actividades artístico-musicales", se impartía en el primer curso del bachillerato con una carga lectiva de dos horas semanales.

Con la incorporación de esta asignatura también lo hicieron los primeros profesores especialistas en la misma, si bien no sería de forma inmediata, lo que ocasionó algunos problemas. Por ejemplo, en el caso de la educación primaria, el principal inconveniente era el hecho de que la asignatura estuviera integrada con otras materias como plástica o educación física, de modo que el profesor de esta área se veía en la necesidad de abarcar una gran cantidad de contenidos de diferente índole. Como explica Lago $(1998)^{3}$ esto suponía una complicación para el desarrollo del área, en tanto que habitualmente el profesor no estaba preparado para abarcar todos estos contenidos, y a menudo se veía obligado a priorizar unos sobre otros, en función de su formación.

En el caso de la educación secundaria la situación no era mucho mejor ya que, dado que en muchas ocasiones no existían grupos suficientes para dotar a un profesor especialista en cada centro, era bastante frecuente que la asignatura fuera impartida por profesorado de otras materias consideradas más o menos afines, o simplemente por profesorado con necesidad de completar su horario lectivo. De este modo, era habitual que el profesor que impartía la asignatura no tuviera una formación musical adecuada para hacerlo. Esta situación empezó a cambiar a partir de los años ochenta, ya que desde el curso 1984/85 empezaron a convocarse oposiciones a profesor de música en bachillerato. Previamente, el Decreto 1194/1982 había equiparado algunos títulos de Conservatorio con los de licenciado universitario, abriendo así la posibilidad a los titulados en música de optar a estas plazas. También en 1984 se aprobaba el título de Licenciado en Musicología, lo que permitiría que la música volviera a estar presente en la universidad ${ }^{4}$.

En 1990 se aprobaba la Ley Orgánica General del Sistema Educativo (LOG$\mathrm{SE})$, que en muchos aspectos siguió vigente hasta entrado el siglo XXI. En esta legislación la música pasó a ocupar un lugar más importante en diferentes etapas de la educación. En concreto, durante la educación primaria formaría parte de las enseñanzas artísticas, junto con la plástica y la dramatización, y durante la educación secundaria se convertiría en un área curricular independiente. Pero además, durante esta ley también se creó la especialidad de Maes-

3. Lago, P. (1998). Análisis del modelo de Formación Permanente del Profesorado de Educación Musical: Valoración de una década de trabajo. Tesis doctoral inédita. Universidad Nacional de Educación a Distancia.

4. Para más información sobre este tema, véase el trabajo de Oriol (2005). 
tro en Educación Musical, dentro de los estudios de magisterio, lo que aseguró que durante la etapa de primaria se impartieran los contenidos de carácter musical, además de que se estableciera una titulación específica para hacerlo.

Otra de las novedades de la LOGSE para el área de música fue la regulación de un bachillerato que permitía compatibilizar los estudios musicales oficiales con la enseñanza secundaria. En concreto, los alumnos que hubieran terminado el tercer ciclo de grado medio de música o danza podían obtener el Título de Bachiller si superaban las materias comunes del bachillerato ${ }^{5}$.

La LOE es la Ley Orgánica de Educación y es la ley vigente en este momento. En lo que se refiere a la materia de música, existen algunas modificaciones en cuanto a la estructura y al planteamiento durante la Educación Secundaria Obligatoria. Al igual que sucedía con la LOGSE, el área de música aparece como una de las materias de los tres primeros cursos de la educación secundaria, si bien no aparece como una de las áreas que se deban impartir en cada uno de estos cursos. Además, puesto que las Comunidades Autónomas pueden hacer modificaciones, ya que el Ministerio determina el $65 \%$ del currículo y el $55 \%$ en las Comunidades con lengua oficial, en algunas regiones la asignatura ha quedado reducida a un solo curso, mientras que en otras se imparte en dos cursos diferentes. En el cuarto curso de la ESO la asignatura de música sigue apareciendo como materia optativa, si bien ya no se aplica la diferenciación entre optativas de modalidad y las que no lo son.

Otra de las novedades de la LOE en materia musical ha sido la inclusión de un nuevo Bachillerato Artístico con un perfil musical. Su denominación es Bachillerato de Artes escénicas, música y danza, y su plan de estudios incluye asignaturas específicas como Historia de la Música, Lenguaje y práctica musical y Análisis Musical I y II, además de Cultura Audiovisual, Literatura Universal, Anatomía aplicada y las asignaturas comunes de bachillerato ${ }^{6}$. La principal diferencia con respecto al bachillerato anterior es que en este caso todas las asignaturas, incluidas las específicas, son impartidas en el centro de secundaria y por el profesorado especialista del mismo.

\section{La música que enseñamos}

Paralelamente a las modificaciones las legislaciones educativas, también el currículo de música ha experimentado una importante evolución. Los motivos son de diferente índole, y por supuesto tienen que ver con los cambios que se han ido produciendo en nuestra sociedad, pero también están relacionados

5. Véase RD 1178/1992 y RD 1179/1992.

6. El currículo de estas asignaturas puede consultarse en el Real Decreto 1467/2007, de 2 de noviembre, por el que establece la estructura del bachillerato y se fijan sus enseñanzas mínimas (BOE 6/11/2007). 
con la formación del propio profesorado que imparte esta materia, que como hemos visto anteriormente, dispone cada vez de una mayor especialización en todas las etapas.

La Ley de 1970 proponía un proyecto educativo desde la perspectiva del modelo tecnológico, que determinaba lo que era necesario enseñar y cómo hacerlo. En lo que se refiere a la asignatura de música, la LGE explicaba que el propósito era ofrecer a los alumnos un conocimiento general del hecho artístico y educar su sensibilidad para la formación estética. En la línea de movimientos como el de la "apreciación musical" desarrollado a mediados del siglo XX en Gran Bretaña, el plan de estudios concedía a la asignatura un carácter eminentemente teórico y completamente vinculado al canon historiográfico occidental. Asimismo, se puntualizaba que el proceso de aprendizaje se debía llevar a cabo a través de audiciones "cuidadosamente preparadas", recomendando la "audición activa" y sugiriendo el uso de medios audiovisuales y de propuestas literarias. La creación y la práctica interpretativa, tanto de carácter vocal como instrumental, quedaban al margen de la asignatura, y eran consideradas como una actividad extraescolar, si bien, la ley sugería vincular al alumno a actividades como el coro escolar y los grupos de baile o de formación rítmica para cursos sucesivos.

En el caso de la LOGSE, la perspectiva psicopedagógica era muy diferente y se sustentaba sobre una concepción constructivista y significativa de la enseñanza y el aprendizaje. Una de las principales novedades del currículo se daba en que los contenidos de todas las materias, entendidos como los medios para lograr los objetivos generales, ya no eran únicamente de tipo conceptual, sino que ahora se contemplaban también los procedimientos y las actitudes. En el área de música los contenidos además se desglosaban en seis diferentes bloques, que muestran la inquietud por aunar la parte más teórica con la práctica musical desde diferentes ámbitos: Expresión vocal y canto, Expresión instrumental, Movimiento y danza, Lenguaje musical, La música en el tiempo y Música y comunicación. Desde la perspectiva pedagógica la asignatura de música ofrecía elementos del modelo tradicional, pero sobre todo incorporaba un acercamiento al modelo progresista, en tanto que por primera vez el currículo perseguía el desarrollo de cualidades como la sensibilidad, la creatividad o la expresividad.

En lo que se refiere a la LOE, una de sus principales novedades ha sido la incorporación de las "competencias básicas", concepto que comenzó a utilizarse a partir del Consejo Europeo de Lisboa, celebrado en marzo de 2000. La LOE las define como aquellas competencias que debe haber desarrollado un joven o una joven al finalizar la enseñanza obligatoria para poder lograr su realización perso-

7. Para consultar las conclusiones del Consejo véase la Web del Parlamento Europeo: http://www.europarl.europa.eu/summits/lis1_es.htm. 
nal, ejercer la ciudadanía activa, incorporarse a la vida adulta de manera satisfactoria y ser capaz de desarrollar un aprendizaje permanente a lo largo de la vida. La Ley también explica que cada una de las áreas contribuye a su desarroIlo, y que aunque no hay una relación directa entre las áreas y las competencias, éstas se alcanzan como consecuencia del trabajo en diferentes materias.

En cuanto a la visión de la música en el currículo de esta legislación, se pueden observar elementos del modelo ecléctico, en tanto que no sólo retoma aspectos del modelo tradicional y del progresista, sino que además plantea algunas novedades. En concreto, hay un interés por mostrar a los alumnos un mayor abanico de estilos y géneros musicales, tanto a través de la audición como de la interpretación o de la creación. Asimismo, el currículo muestra una importante influencia de diferentes disciplinas afines como la sociología de la música, la etnomusicología o la psicología cognitiva, y además aporta la visión de una educación musical para una sociedad plural.

Por otra parte, los bloques de contenido de la asignatura no son iguales para toda la etapa. Como puede verse en la Tabla 2 los bloques, diferentes de los establecidos por la LOGSE, pasan a ser cuatro en los cursos de primero a tercero, y tres en el caso de cuarto.

Tabla 2. Relación entre los bloques de contenido de la LOGSE y la LOE.

\begin{tabular}{|l|l|l|}
\hline $\begin{array}{c}\text { BLOQUES DE CONTENIDO } \\
\text { LOGSE }\left(\mathbf{1}^{\mathbf{0}} \mathbf{A} \mathbf{4}^{\mathbf{0}}\right)\end{array}$ & $\begin{array}{c}\text { BLOQUES DE CONTENIDO } \\
\mathbf{L O E}\left(\mathbf{1}^{\mathbf{0}} \mathbf{A} \mathbf{3}^{\mathbf{0}}\right)\end{array}$ & $\begin{array}{c}\text { BLOQUES DE CONTENIDO } \\
\mathbf{L O E}\left(\mathbf{4}^{\mathbf{o}}\right)\end{array}$ \\
\hline -Expresión vocal y canto & -Escucha & -Audición y referentes musicales \\
\hline -Expresión instrumental & -Interpretación & -La práctica musical \\
\hline -Movimiento y danza & -Creación & -Música y tecnologías \\
\hline -Lenguaje musical & -Contextos musicales & \\
\hline -La música en el tiempo & & \\
\hline -Música y comunicación & & \\
\hline
\end{tabular}

El currículo vigente plantea la asignatura de música en la educación general desde una importante variedad de aprendizajes, recursos y valores. Esta perspectiva es un reflejo de la transformación de la música en la sociedad, pero también de otros cambios sociales, culturales y tecnológicos más profundos relacionados con la globalización, la demografía, la multiculturalidad, la igualdad de género o la incorporación de las nuevas tecnologías a la vida cotidiana. Lógicamente, la educación musical del siglo XXI debe contribuir a que el alumnado comprenda y asimile todas estas transformaciones.

Una de las principales novedades de la educación musical actual es el interés por la educación multicultural e intercultural, que son dos de los términos que se han venido utilizando para describir la perspectiva pedagógica orienta- 
da a conocer, comprender y promover la tolerancia entre las diferentes culturas en una sociedad cada vez más diversa ${ }^{8}$. La creación de un currículo intercultural es la consecuencia de una situación social y política que reconoce el pluralismo cultural y los beneficios de esta diversidad, un sistema educativo que la integra y unos medios de comunicación que nos facilitan el acercamiento a las diferentes culturas.

Desde la perspectiva pedagógica, educadores y etnomusicólogos empezaron a colaborar en busca de los materiales musicales y de los métodos más adecuados para la enseñanza de las músicas del mundo. Sin duda, este es uno de los puntos que ha generado los debates más interesantes y que nos muestra que todavía queda mucho por hacer en torno a este tema.

En España el origen de educación musical intercultural se remonta a los años noventa con la LOGSE, que expandió el repertorio musical más allá de la música clásica occidental. Sin embargo, el verdadero impulso para esta educación ha Ilegado con la LOE, en donde las músicas de diferentes culturas no sólo se mencionan de forma explícita, sino que además se especifica su importancia como fuente de conocimiento y para el enriquecimiento intercultural.

Pero sin duda, el aspecto que más ha evolucionado entre ambas leyes ha sido el tratamiento de las Tecnologías de la Información y la Comunicación (TIC), que lógicamente es el resultado de la importancia de las mismas en la sociedad actual. En concreto, la LOGSE mencionaba los audiovisuales como una fuente de información; por su parte, la LOE manifiesta la importancia del desarrollo tecnológico para la modificación de los referentes musicales de una sociedad, y subraya su repercusión en la aparición de nuevos cauces para la interpretación y la creación. Además, esta legislación contempla por primera vez un objetivo general de música relacionado con las TIC:

Conocer y utilizar diferentes medios audiovisuales y tecnologías de la información y la comunicación como recursos para la producción musical, valorando su contribución a las distintas actividades musicales y al aprendizaje autónomo de la música.

Desde los años noventa diferentes investigadores en educación, tanto del panorama nacional como internacional, han llevado a cabo numerosas propuestas sobre el uso de las nuevas tecnologías en la educación musical. Lógicamente, enseñar música en el nuevo siglo exige hacer un uso eficaz de las TIC, en tanto que contribuyen a mejorar la docencia de música desde diferentes ámbitos.

8. El término multicultural es utilizado especialmente en el ámbito norteamericano, mientras que el término intercultural ha sido más utilizado en Europa, en donde se matiza además que también implica la interrelación entre individuos de diferentes culturas y el aprendizaje mutuo entre todas ellas (Volk 1998). 


\section{A modo de conclusión}

La enseñanza de música en nuestro país tiene una historia relativamente reciente. Hasta los años setenta estaba vinculada al ámbito privado, de modo que la formación musical no era algo frecuente entre la población. En los años setenta, la primera legislación que la incorporó a la enseñanza obligatoria lo hizo ciñéndola casi de forma exclusiva al conocimiento teórico de la historia de la música. Sin embargo, durante los años noventa el currículo de la LOGSE buscó aunar la parte teórica y la práctica, algo que la siguiente legislación ha seguido manteniendo. Además de su importante contribución al desarrollo neurológico y cognitivo, el hecho de que la música sea un elemento presente en todas las sociedades conocidas puede ayudar al individuo a conocer mejor la sociedad que le rodea. Sin embargo, al igual que sucede en otros países, la asignatura no es considerada una materia troncal y no está sujeta a evaluaciones internacionales. Por ese motivo no se suele valorar de forma objetiva su aportación a la educación o, siguiendo a Regelski (2009), su contribución para "marcar una diferencia" en la vida de quienes tienen la oportunidad de estudiarla.

\section{Coda}

A la espera de la publicación de una nueva ley educativa en España, que va a llevar el nombre de LOMCE (Ley Orgánica para la Mejora de la Calidad en la Educación), los dos Anteproyectos publicados en septiembre y diciembre de 2012, respectivamente, han planteado la preocupación por los resultados obtenidos por el alumnado en las pruebas de evaluación internacionales PISA (Programme for International Student Assessment) en materias como comprensión lectora, competencia matemática y competencia científica. Uno de los principales objetivos que se plantea esta ley es mejorar los resultados del alumnado en las pruebas internacionales, y el primer anteproyecto explicaba que "el nivel educativo de un país determina su capacidad de competir con éxito en la arena internacional". Para ello, la nueva Ley aboga por conceder a las materias troncales una carga lectiva superior.

\section{Bibliografía}

GARDNER, H. Frames of mind: The theory of multiple intelligences. New York: BasicBooks, 1983.

HALLAM, S. Music psychology in education. Londres: Institute of Education, 2006. HODGES, D. A. "The musical brain". En G. McPherson (ed.), The child as musician. A handbook of musical development. New York: Oxford University Press, 2006, pp. 51-68.

LAGO, P. Análisis del modelo de Formación Permanente del Profesorado de Educación Musical: Valoración de una década de trabajo. Tesis doctoral inédita. Universidad Nacional de Educación a Distancia, 1998. 
ORIOL, N. "La música en las enseñanzas de régimen general en España y su evolución en el siglo XX y comienzos del XXI". Revista electrónica de Léeme. (Lista Electrónica Europea de Música en la Educación) 16. En http://musica.rediris.es/leeme/index.html.

RAUSCHER, F. H., SHAW, G. L. y KY, K. N. "Music and spatial task performance". Nature 365, 1993, p. 611.

REGELSKI, T. A. (2009). "La música y la educación musical: Teoría y práctica para 'marcar una diferencia'". En D. K. Lines (comp.), La educación musical para el nuevo milenio. Madrid: Morata, 2009, pp. 21-47.

ROSS, A. Escucha esto. Barcelona: Seix Barral, 2012.

SCHLAUGH, G. "The brain of musicians". En I. Peretz y R. J. A. Zatorre (eds.), The cognitive neuroscience of music. Oxford: Oxford University Press, 2003, pp. 366-381.

SCHELLENBERG, E. G. "Music lessons enhance IQ". Psychological Science 15, 2004, pp. 511-514.

—, "Exposure to music: The truth about the consequences". En G. McPherson (ed.), The child as musician. Oxford: Oxford University Press, 2005, pp. 111-134.

STEWART, L. et al. "Brain changes after learning to read and play music". Neurolmage 20, 2003, 71-83.

VOLK, T. W. Music, Education and Multiculturalism. Oxford: Oxford University Press, 1998. 